\title{
Gestión del talento humano: Diagnóstico y sintomatología de trastornos musculoesqueléticos evidenciados a través del Cuestionario Nórdico de Kuorinka
}

\section{Management of human resources: Diagnosis and symptoms of musculoskeletal disorders evidenced through the Nordic Kuorinka Questionnaire}

Sebastián Ricardo Castro García

https://orcid.org/0000-0002-3393-979X

Secretaría de Educación Superior, Ciencia, Tecnología e Innovación, SENESCYT, Ecuador

Edison Daniel Yandún Burbano

iD https://orcid.org/0000-0001-7871-1417

Universidad Internacional del Ecuador, Ecuador

Luis Fernando Freire Constante

(iD https://orcid.org/0000-0002-9936-3041

Universidad Técnica Particular de Loja, Ecuador

Mayra Gisella Albán Álvarez

(iD https://orcid.org/0000-0002-8928-4251

Consultor Independiente, Ecuador

Autor para correspondencia: sebastiancastrog@outlook.com; danielyandun@ hotmail.com; 1freire@systemsvt.com; albanmayra@ hotmail.com

Fecha de recepción: 28 de septiembre de 2020 - Fecha de aceptación: 18 de diciembre de 2020

\section{Resumen}

La presente investigación es un estudio descriptivo no experimental de corte transversal, cuyo propósito es evidenciar el diagnóstico y sintomatología de posibles trastornos musculoesqueléticos de trabajadores administrativos a través de la utilización del Cuestionario Nórdico Estandarizado de Síntomas Musculoesqueléticos de Kuorinka, en sus actividades laborales. El objetivo del presente estudio es contribuir a la concienciación de las empresas en materia de gestión del talento humano en la línea de prevención de riesgos laborales, diagnosticando sintomatologías de posibles enfermedades ocupacionales desde la percepción del trabajador a través de herramientas validadas se puede prevenir las mismas de tal forma que en las organizaciones podamos tener trabajadores sanos y productivos que cumplan los objetivos organizacionales, además de evitarse costos por incumplimiento de gestión de seguridad y salud ocupacional, y obviamente evitar problemas sociales de personal enfermo o con posibles discapacidades. La población que se evidencia en la 
presente investigación es de doscientas sesenta y ocho personas $(\mathrm{N}=268)$ que realizan labores administrativas pertenecientes a diez empresas ecuatorianas. Los resultados reflejan cierta similitud de percepción en los géneros masculino $\left(n_{1}=104\right)$ y femenino $\left(n_{2}=164\right)$ que sobrepasan el 75\% de las muestras analizadas, siendo los más relevantes mano muñeca derecha e izquierda, columna lumbar y cuello. Estos datos obtenidos son el reflejo de la percepción ante posibles trastornos musculoesqueléticos y especialmente en el rango de edad entre 35 a 44 años, ante lo cual los responsables de talento humano y seguridad y salud ocupacional disponen de una información basal para poder plantearse medidas de prevención y control ergonómicos en puestos administrativos.

Palabras claves: cuestionario nórdico; ergonomía; músculo esquelético; talento humano.

\begin{abstract}
The present investigation is a non-experimental descriptive cross-sectional study, the purpose of which is to demonstrate the diagnosis and symptomatology of possible musculoskeletal disorders of administrative workers through the use of the Nordic Standardized Questionnaire of Musculoskeletal Symptoms of Kuorinka, in their work activities. The objective of this study is to contribute to the awareness of companies in the prevention of occupational hazards, diagnosing symptoms of possible occupational diseases from the perception of the worker through validated tools, they can be prevented in such a way that in organizations we can have healthy and productive workers who meet organizational objectives, in addition to avoiding costs for breach of management in occupational health and safety, and obviously avoid social problems of sick or disabled personnel. The sample that is evidenced in this investigation is two hundred and sixtyeight people $(\mathrm{N}=268$ ) who carry out administrative tasks belonging to ten Ecuadorian companies. The results reflect a certain similarity of perception in the male $\left(\mathrm{n}_{1}=104\right)$ and female $\left(\mathrm{n}_{2}=164\right)$ genres, which exceed $75 \%$ of the samples analyzed, the most relevant being the right and left wrist, lumbar spine and neck. These data obtained are a reflection of the perception of possible musculoskeletal disorders and especially in the age range between 35 to 44 years of age, before which those responsible for occupational safety and health have baseline information to be able to consider prevention and ergonomic control in administrative positions.
\end{abstract}

Keywords: nordic questionnaire; ergonomics; skeletal muscle; human resources.

\title{
Introducción
}

Los trastornos musculoesqueléticos relacionados con el trabajo son muy comunes en una población. (Widanarko et al., 2014). Estudios demuestran que estos son una de las principales causas de las enfermedades laborales por lo que se buscan estrategias conjuntas entre las partes interesadas para mejorar y modificar prácticas del trabajo que puedan afectar a las personas. (Bosman et al., 2020). Los trastornos musculoesqueléticos se pueden caracterizar por ser multifactoriales por lo que engloban a componentes psicosociales, físicos, individuales y ocupacionales. (Rasha et al., 2019). Aquellos que son relacionados con el trabajo se presentan cuando existe una discordancia entre la capacidad física del cuerpo humano versus los requisitos físicos de la tarea. (Orhan y Memon, 2019). 
Datos de la Agencia Europea para la Seguridad y Salud en el Trabajo demuestran que los trastornos musculoesqueléticos son la cuarta afección de los países europeos de los cuales el $25 \%$ manifiesta que sufren dolores de espalda y $23 \%$ son dolores musculares. El Instituto Nacional de Seguridad e Higiene del Trabajo en la sexta encuesta Nacional de Condiciones del Trabajo (INSHT, 2017) recopila que el 74\% de la población trabajadora presenta molestias musculoesqueléticas producto de malas posturas o sobre esfuerzos a causa de su actividad laboral y que la espalda baja y cuello son las partes más comprometidas. (Arenas-Ortiz y CantúGómez, 2013).

Mil setecientos millones de personas en todo el mundo son afectadas por trastornos musculoesqueléticos por lo que representa el cuarto mayor impacto en la salud general de la población mundial. (Nazish et al., 2020). Estos costos son altos independientemente de la situación económica del país. (Qureshi et al., 2019).

El dolor a causa de estas afecciones se presenta tanto en países en desarrollo como en subdesarrollo con datos de prevalencia que va desde el once al sesenta por ciento y que la prevalencia de dolor musculoesquelético entre las mujeres presentó un mayor valor en comparación con los datos de los hombres. (Nazish et al., 2020). El malestar esta originado por afecciones inflamatorias y degenerativas que afectan directamente a tendones, músculos nervios articulaciones y huesos, afectando negativamente la calidad de vida. (Alaca et al., 2019).

Trabajadores operativos o administrativos pueden desarrollar estos trastornos por sus actividades habituales de trabajo (Kuorinka et al., 1987). La actividad laboral administrativa ha generado problemas de salud laboral, el uso de laptops y desktops sin ningún criterio ocupacional ha causado lesiones en manos, brazos, hombros cuello, espalda baja, muñeca con importantes síntomas de rigidez y dolor. (Iti et al., 2016). La parte baja de la espalda puede ser en algunos casos la más predominante (Rasha et al., 2019). (Zamanian et al., 2017). El dolor lumbar compromete la capacidad funcional y el no tener un instrumento de validación limita las actividades preventivas. (Oliveira et al., 2017). (Gómez Rodríguez et al., 2020).

Ante esta situación desde el punto de vista laboral es necesario identificar una herramienta ergonómica que permita diagnosticar la magnitud de los trastornos musculoesqueléticos que podrían presentarse producto de las actividades laborales. (Kwaku Essien et al., 2016).

El Cuestionario Nórdico es una herramienta estandarizada y uno de los más usados en el análisis ergonómico (Namnik et al., 2016)., que fue publicada en el año 1987 y es utilizada como encuesta auto aplicada o como entrevista para la obtención de datos de sintomatología previa a la aparición de una enfermedad declarada por lo que facilita la toma de acciones preventivas. (Martínez y Alvarado Muñoz, 2017). Esta herramienta ergonómica se creó para responder a lo siguiente. “¿Se producen problemas musculoesqueléticos en una población determinada? y, en caso afirmativo, ¿En qué partes del cuerpo se localizan?”. (Kuorinka et al., 1987).

El cuestionario Nórdico de Kuorinka es muy usado en el análisis y detección de síntomas musculo esqueléticos, está enfocado en la gestión de prevención de riesgos ergonómicos con el fin de detectar la existencia de los primeros síntomas de alguna afectación, pero sin que se haya 
manifestado una enfermedad laboral y aunque es un método relativamente antiguo su valor técnico radica en la información entregada la cual permite estimar el nivel de riesgo de manera proactiva por lo que la intervención puede ser inmediata. (Dimate et al., 2017) Se ha encontrado algunos estudios de importancia que han utilizado este cuestionario de evaluación. Un estudio relevante fue realizado Chile con el nombre de "Validación del Cuestionario Nórdico Estandarizado de Síntomas Musculoesqueléticos para la población trabajadora chilena, adicionando una escala de dolor" en una muestra de 114 trabajadores de 6 rubros económicos se realizó la validación del cuestionario a una submuestra de 54 trabajadores.(Martínez y Alvarado Muñoz, 2017). En Venezuela se utilizó el método para determinar "Síntomas Músculo Esqueléticos en Trabajadores de una Empresa de Construcción Civil" En donde se buscaba determinar la prevalencia de síntomas músculo esqueléticos y su relación con las características sociodemográficas y ocupacionales en trabajadores de una empresa de construcción civil en 89 trabajadores masculinos. (Bellorín et al., 2007) En Colombia se realizó un estudio de percepción de molestias musculoesqueléticas en nueve regiones corporales utilizando el Cuestionario Nórdico tomando una muestra de 302 trabajadores. (Mendinueta Martínez \& Herazo Beltrán, 2014)

A través de este estudio se buscó determinar la percepción, diagnóstico y sintomatología de posibles trastornos musculoesqueléticos, a través del Cuestionario Nórdico Estandarizado de Síntomas Musculoesqueléticos de Kuorinka, para tener un nivel basal de actuación en el medio ambiente laboral y poder plantear las medidas de prevención ergonómicas de los trabajadores administrativos expuestos.

\section{Materiales y métodos}

El diseño y fuente de información de esta investigación se basa en un estudio descriptivo no experimental de corte transversal, sobre las causas y sintomatologías de trastornos musculoesqueléticos que tienen los trabajadores administrativos de diez empresas ecuatorianas, con una población de doscientas sesenta y ocho personas $(\mathrm{N}=268)$. La obtención de los datos a la población indicada se lo realizó a través del Cuestionario Nórdico Estandarizado de Síntomas Musculoesqueléticos de Kuorinka.

De acuerdo a las estrategias para determinar la existencia de riesgos que manifiesta: "la persona que mejor conoce el trabajo es quien lo realiza. Muy a menudo, el punto de vista de los gerentes acerca de las causas de los problemas puede distar mucho del de sus empleados. Pida siempre la opinión de sus empleados". (OMS, 2004), es por esta razón que se eligió utilizar el Cuestionario Nórdico Estandarizado de Síntomas Musculoesqueléticos de Kuorinka, ya que determina en once preguntas la percepción y la sintomatología del trabajador al que están expuestos en su actividad laboral administrativa.

La primera fase de la presente investigación se solicitó la autorización a los departamentos de talento humano de las diez empresas analizadas para poder estimar la muestra del presente estudio de doscientas sesenta y ocho personas $(\mathrm{N}=268)$, teniendo en cuenta como criterio de inclusión el que realicen su actividad laboral en oficinas y con un tiempo de utilización de los computadores o laptops de cuatro horas o más, y como criterio de exclusión a 
aquellos colaboradores que no cumplan su actividad laboral de forma constante en las oficinas y la utilización de equipos informáticos sea inferior a cuatro horas. En la segunda fase se procedió a enviar la encuesta a través de Google Forms con una aceptación y respuesta del cien por ciento $(\mathrm{N}=268)$, teniendo en consideración la confidencialidad de los trabajadores para sus empresas. En la tercera fase del presente estudio se procedió a tabular los datos obtenidos de los doscientos sesenta y ocho cuestionarios recibidos; y, en la cuarta y última fase del estudio se realizó el análisis, discusión y recomendaciones de los resultados obtenidos.

Al ser un estudio no experimental por haber aplicado el Cuestionario Nórdico Estandarizado de Síntomas Musculoesqueléticos de Kuorinka, las variables analizadas en la presente investigación son dos que se componen de la siguiente manera: variable sociodemográfica en la cual se identifica la edad y el género, y la segunda variable se compone de cinco segmentos corporales que define el cuestionario siendo los siguientes: cuello, hombro izquierdo y derecho, columna dorsal y lumbar, codo o antebrazo; y, muñeca o mano. Cada uno de los componentes de la variable del cuestionario utilizado tiene como característica fundamental la frecuencia e intensidad de la percepción o posible trastorno musculoesquelético de los trabajadores objeto de la muestra.

Las herramientas utilizadas en la presente investigación fueron las siguientes: Software aplicación de encuesta: Google Forms. Software tabulación de datos: Microsoft Excel 2019. Programa estadístico informático: Statistical Package for the Social Sciences (SPSS). Software redacción del documento: Microsoft Word 2019. Gestor bibliográfico: Mendeley Reference Manager.

\section{Resultados}

Se ha tabulado un total de doscientos sesenta y ocho cuestionarios, realizados a trabajadores administrativos de diez empresas ecuatorianas, cumpliendo estrictamente el criterio de inclusión de utilizar como mínimo el computador y/o laptop por un lapso de cuatro horas y que su actividad lo realiza en las instalaciones de las empresas analizadas.

En el análisis de la primera variable sociodemográfica (Tabla 1), que corresponde al género se evidencia que el $61.2 \%(\mathrm{~N}=268 ; 164)$ son de género femenino y el $38.8 \%(\mathrm{~N}=268$; 104) pertenecen al género masculino, en el análisis de edades se observa que el rango que contiene mayor población es el de 35 a 44 años con 39.6\% ( $\mathrm{N}=268 ; 106)$, seguido del $31.3 \%$ $(\mathrm{N}=268 ; 84)$ que corresponde a una edad entre 25 a 34 años, las personas $>45$ corresponden a un $15.7 \%(\mathrm{~N}=268 ; 42)$; y, el rango de edad más bajo alcanza $13.4 \%(\mathrm{~N}=268 ; 36)$ correspondiente a las personas < de 25 años. 
Tabla 1

Resultados Cuestionario Nórdico Estandarizado de Síntomas Musculoesqueléticos de Kuorinka: Variable Sociodemográfica.

\begin{tabular}{lcc}
\hline \multicolumn{1}{c}{ Variable } & Variable sociodemográfica & \\
\hline 1. Género & Frecuencia & Porcentaje \\
\hline Masculino & & \\
Femenino & 104 & $38.8 \%$ \\
Otros & 164 & $61.2 \%$ \\
& 0 & $0 \%$ \\
\hline 2. Edad & & \\
Menor a 25 años & 36 & $13.4 \%$ \\
De 25 a 34 años & 84 & $31.3 \%$ \\
De 35 a 45 años & 106 & $39.6 \%$ \\
45 años en adelante & 42 & $15.7 \%$ \\
& & \\
\hline \multicolumn{1}{c}{$\boldsymbol{N}=\mathbf{2 6 8}$} & & \\
\hline
\end{tabular}

Fuente: Autores.

En el análisis de la segunda variable de los cinco segmentos corporales que indica el Cuestionario Nórdico Estandarizado de Síntomas Musculoesqueléticos de Kuorinka, para evidenciar los resultados se ha dividido por género, es así que para el género masculino tenemos una muestra de análisis de ciento cuatro personas $\left(\mathrm{n}_{1}=104\right)$, y para el género femenino la muestra es de ciento sesenta y cuatro $\left(\mathrm{n}_{2}=164\right)$.

Las evidencias del género masculino (Tabla 2), en el primer segmento corporal relacionado al cuello, la percepción de un posible trastorno musculoesquelético alcanza el 36.5\% $\left(\mathrm{n}_{1}=104 ; 38\right)$ en el rango de edad de 35 a 44 años de edad, los datos obtenidos de percepción de molestias del segundo segmento corporal hombros se evidencia que el hombro izquierdo no superan el 10\%, siendo el más representativo el 8.7\% ( $\left.\mathrm{n}_{1}=104 ; 9\right)$ en el rango de 25 a 34 años, los posibles trastornos de hombro derecho alcanzan su máximo porcentaje en el rango de edad de 35 a 44 años con el 12.5\% $\left(n_{1}=104 ; 13\right)$ de la muestra. El tercer segmento corporal se clasifica en columna dorsal y lumbar, siendo el dato más relevante obtenido en columna dorsal del cuestionario con el $21.2 \%\left(\mathrm{n}_{1}=104 ; 22\right)$ en la edad de 35 a 44 años, evidenciando que el porcentaje más alto en este rango de edad en lo referente a la columna lumbar es del $36.5 \%$ $\left(\mathrm{n}_{1}=104 ; 38\right)$. El cuarto segmento corporal perteneciente a codo/antebrazo también se deriva en izquierdo y derecho, los datos tabulados para codo/antebrazo izquierdo es el porcentaje más alto obtenido alcanzando el $11.5 \%\left(\mathrm{n}_{1}=104 ; 12\right)$ en el rango de edad de 35 a 44 años de edad; y, también el dato más representativo alcanzado se da en este mismo grupo para codo/antebrazo derecho con el $14.4 \%\left(n_{1}=104 ; 15\right)$, seguido de un $13.5 \%\left(n_{1}=104 ; 14\right)$ en personas $>$ de 45 años. El quinto y último segmento corporal que evalúa el cuestionario es mano/muñeca izquierda y derecha, donde se refleja que los porcentajes más altos obtenidos están en el rango de edad de 35 a 44 años alcanzando un $34.6 \%\left(n_{1}=104 ; 36\right)$ para mano/muñeca izquierda; y, para mano/muñeca derecha el $38.5 \%\left(\mathrm{n}_{1}=104 ; 40\right)$. 


\section{Tabla 2}

Resultados Cuestionario Nórdico Estandarizado de Síntomas Musculoesqueléticos de Kuorinka: Percepción de síntomas musculoesqueléticos por segmento corporal, grupos de edad del género masculino.

\begin{tabular}{|c|c|c|c|c|c|c|c|c|c|c|c|c|c|c|c|c|}
\hline \multicolumn{17}{|c|}{ Percepción de síntomas musculo esqueléticos por segmento corporal, grupos de edad del género masculino } \\
\hline \multirow{3}{*}{$\begin{array}{l}\text { Segmento } \\
\text { Corporal }\end{array}$} & \multicolumn{4}{|c|}{$<25$ años } & \multicolumn{4}{|c|}{$25-34$ años } & \multicolumn{4}{|c|}{35 - 44 años } & \multicolumn{4}{|c|}{$>45$ años } \\
\hline & & & 12 & & & & & & & $n=$ & & & & $n=$ & & \\
\hline & $* \mathrm{Si}$ & $\%$ & $*$ No & $\%$ & $\mathrm{Si}$ & $\%$ & No & $\%$ & $\mathrm{Si}$ & $\%$ & No & $\%$ & $\mathrm{Si}$ & $\%$ & No & $\%$ \\
\hline Cuello & 7 & $6.7 \%$ & 5 & $4.8 \%$ & 32 & $30.8 \%$ & 3 & $2.9 \%$ & 38 & $36.5 \%$ & 3 & $2.9 \%$ & 14 & $13.5 \%$ & 2 & $1.9 \%$ \\
\hline $\begin{array}{l}\text { Hombro - } \\
\text { Izquierdo }\end{array}$ & 0 & $0.0 \%$ & 12 & $11.5 \%$ & 9 & $8.7 \%$ & 26 & $25.0 \%$ & 7 & $6.7 \%$ & 34 & $32.7 \%$ & 5 & $4.8 \%$ & 11 & $10.6 \%$ \\
\hline $\begin{array}{l}\text { Hombro - } \\
\text { Derecho }\end{array}$ & 0 & $0.0 \%$ & 12 & $11.5 \%$ & 11 & $10.6 \%$ & 24 & $23.1 \%$ & 13 & $12.5 \%$ & 28 & $26.9 \%$ & 8 & $7.7 \%$ & 8 & $7.7 \%$ \\
\hline $\begin{array}{l}\text { Columna } \\
\text { Dorsal }\end{array}$ & 1 & $1.0 \%$ & 11 & $10.6 \%$ & 12 & $11.5 \%$ & 23 & $22.1 \%$ & 22 & $21.2 \%$ & 19 & $18.3 \%$ & 13 & $12.5 \%$ & 3 & $2.9 \%$ \\
\hline $\begin{array}{l}\text { Columna } \\
\text { Lumbar }\end{array}$ & 9 & $8.7 \%$ & 3 & $2.9 \%$ & 33 & $31.7 \%$ & 2 & $1.9 \%$ & 38 & $36.5 \%$ & 3 & $2.9 \%$ & 15 & $14.4 \%$ & 1 & $1.0 \%$ \\
\hline $\begin{array}{l}\text { Codo / } \\
\text { Antebrazo - } \\
\text { Izquierda }\end{array}$ & 0 & $0.0 \%$ & 12 & $11.5 \%$ & 3 & $2.9 \%$ & 32 & $30.8 \%$ & 12 & $11.5 \%$ & 29 & $27.9 \%$ & 9 & $8.7 \%$ & 7 & $6.7 \%$ \\
\hline $\begin{array}{l}\text { Codo / } \\
\text { Antebrazo - } \\
\text { Derecha }\end{array}$ & 0 & $0.0 \%$ & 12 & $11.5 \%$ & 8 & $7.7 \%$ & 27 & $26.0 \%$ & 15 & $14.4 \%$ & 26 & $25.0 \%$ & 14 & $13.5 \%$ & 2 & $1.9 \%$ \\
\hline $\begin{array}{l}\text { Mano / } \\
\text { Muñeca - } \\
\text { Izquierda }\end{array}$ & 5 & $4.8 \%$ & 7 & $6.7 \%$ & 25 & $24.0 \%$ & 10 & $9.6 \%$ & 36 & $34.6 \%$ & 5 & $4.8 \%$ & 13 & $12.5 \%$ & 3 & $2.9 \%$ \\
\hline $\begin{array}{l}\text { Mano / } \\
\text { Muñeca - } \\
\text { Derecha }\end{array}$ & 11 & $10.6 \%$ & 1 & $1.0 \%$ & 34 & $32.7 \%$ & 1 & $1.0 \%$ & 40 & $38.5 \%$ & 1 & $1.0 \%$ & 14 & $13.5 \%$ & 2 & $1.9 \%$ \\
\hline
\end{tabular}

$n_{1}=104$

Descripción: *Si - Presenta sintomatología.

*No - No presenta sintomatología.

Fuente: Autores.

Los datos obtenidos de los posibles problemas musculoesqueléticos (Figura 1), en el género masculino se evidencia en forma global, siendo los rangos de edad entre 25-34 años y 3544 años los más representativos. El rango de edad de 35 a 44 años presenta porcentajes más altos siendo las dolencias más prevalentes en: mano muñeca derecha $38.5 \%$, cuello y columna lumbar $36.5 \%$, mano muñeca izquierda $34.6 \%$, el segundo rango de edad con dolencias más prevalentes es el de 25-34 años en: mano muñeca derecha $32.7 \%$, columna lumbar $31.7 \%$, cuello $30.8 \%$ y mano muñeca izquierda $24 \%$. 


\section{Figura 1}

Percepción de sintomatología musculoesquelética - Género Masculino.

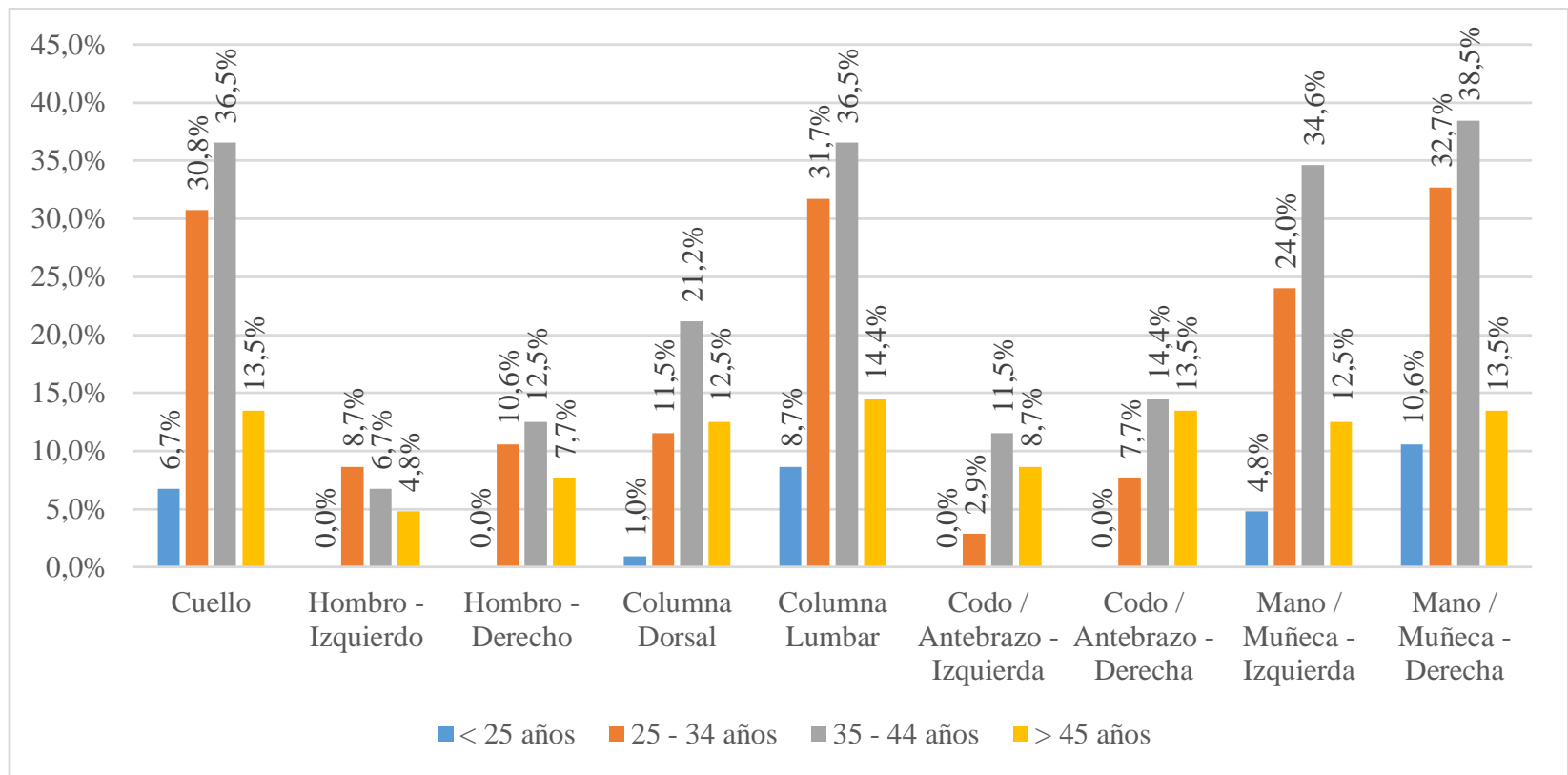

Fuente: Autores.

La muestra para el género femenino es de ciento sesenta y cuatro $\left(\mathrm{n}_{2}=164\right)$, los datos obtenidos (Tabla 3), evidencian en el primer segmento corporal qué, el cuello es el porcentaje más alto con el 38.4\% $\left(\mathrm{n}_{2}=164 ; 63\right)$ en el rango de edad de 35 a 44 años. El segundo ítem evaluado referente a hombro izquierdo, el porcentaje más representativo es el $7.9 \%\left(\mathrm{n}_{2}=164 ; 13\right)$ en el rango de edad de $>45$ años, en cambio para el hombro derecho el porcentaje más alto obtenido es el rango de 35 a 44 años alcanzando el $25.6 \%\left(n_{2}=164 ; 42\right)$. La percepción de trastornos musculoesqueléticos en columna dorsal evidencia que el $23.8 \%\left(\mathrm{n}_{2}=164 ; 39\right) \mathrm{se}$ manifiesta en mujeres de 35 a 44 años y en columna lumbar alcanza el $37.8 \%\left(n_{2}=164 ; 62\right)$ también en el mismo rango de edad. El cuarto segmento corporal en el rango de edad de 35 a 44 años evidencia los porcentajes más altos, para codo/antebrazo izquierdo alcanza el $17.1 \%$ $\left(\mathrm{n}_{2}=164 ; 28\right)$; $\mathrm{y}$, para codo/antebrazo derecho se obtuvo el $18.9 \%\left(\mathrm{n}_{2}=164 ; 31\right)$. El quinto segmento corporal en el rango de edad de 35 a 44 años presenta los porcentajes más altos obtenidos, para mano/muñeca izquierda se obtuvo el 35.4\% $\left(\mathrm{n}_{2}=164 ; 58\right)$; y, para mano/muñeca derecha el $39 \%\left(\mathrm{n}_{2}=164 ; 64\right)$ respectivamente. 


\section{Tabla 3}

Resultados Cuestionario Nórdico Estandarizado de Síntomas Musculoesqueléticos de Kuorinka: Percepción de síntomas musculoesqueléticos por segmento corporal, grupos de edad del género femenino.

\begin{tabular}{|c|c|c|c|c|c|c|c|c|c|c|c|c|c|c|c|c|}
\hline \multicolumn{17}{|c|}{ Percepción de síntomas musculo esqueléticos por segmento corporal, grupos de edad del género femenino } \\
\hline \multirow{3}{*}{$\begin{array}{l}\text { Segmento } \\
\text { Corporal }\end{array}$} & \multicolumn{4}{|c|}{$<25$ años } & \multicolumn{4}{|c|}{25 - 34 años } & \multicolumn{4}{|c|}{35 - 44 años } & \multicolumn{4}{|c|}{$>45$ años } \\
\hline & & $n=$ & 24 & & & & $=49$ & & & & $=65$ & & & & 26 & \\
\hline & $* \mathbf{S i}$ & $\%$ & $*$ No & $\%$ & Si & $\%$ & No & $\%$ & $\mathbf{S i}$ & $\%$ & No & $\%$ & $\mathbf{S i}$ & $\%$ & No & $\%$ \\
\hline Cuello & 13 & $7.9 \%$ & 11 & $6.7 \%$ & 46 & $28.0 \%$ & 3 & $1.8 \%$ & 63 & $38.4 \%$ & 2 & $1.2 \%$ & 25 & $15.2 \%$ & 1 & $0.6 \%$ \\
\hline $\begin{array}{l}\text { Hombro - } \\
\text { Izquierdo }\end{array}$ & 1 & $0.6 \%$ & 23 & $14.0 \%$ & 12 & $7.3 \%$ & 37 & $22.6 \%$ & 11 & $6.7 \%$ & 54 & $32.9 \%$ & 13 & $7.9 \%$ & 13 & $7.9 \%$ \\
\hline $\begin{array}{l}\text { Hombro - } \\
\text { Derecho }\end{array}$ & 3 & $1.8 \%$ & 21 & $12.8 \%$ & 16 & $9.8 \%$ & 33 & $20.1 \%$ & 42 & $25.6 \%$ & 23 & $14.0 \%$ & 19 & $11.6 \%$ & 7 & $4.3 \%$ \\
\hline $\begin{array}{l}\text { Columna } \\
\text { Dorsal }\end{array}$ & 2 & $1.2 \%$ & 22 & $13.4 \%$ & 14 & $8.5 \%$ & 35 & $21.3 \%$ & 39 & $23.8 \%$ & 26 & $15.9 \%$ & 17 & $10.4 \%$ & 9 & $5.5 \%$ \\
\hline $\begin{array}{l}\text { Columna } \\
\text { Lumbar } \\
\text { Codo / }\end{array}$ & 18 & $11.0 \%$ & 6 & $3.7 \%$ & 44 & $26.8 \%$ & 5 & $3.0 \%$ & 62 & $37.8 \%$ & 3 & $1.8 \%$ & 24 & $14.6 \%$ & 2 & $1.2 \%$ \\
\hline $\begin{array}{l}\text { Antebrazo } \\
\text { - Izquierda }\end{array}$ & 2 & $1.2 \%$ & 22 & $13.4 \%$ & 6 & $3.7 \%$ & 43 & $26.2 \%$ & 28 & $17.1 \%$ & 37 & $22.6 \%$ & 11 & $6.7 \%$ & 15 & $9.1 \%$ \\
\hline $\begin{array}{l}\text { Codo / } \\
\text { Antebrazo } \\
\text { - Derecha }\end{array}$ & 1 & $0.6 \%$ & 23 & $14.0 \%$ & 13 & $7.9 \%$ & 36 & $22.0 \%$ & 31 & $18.9 \%$ & 34 & $20.7 \%$ & 18 & $11.0 \%$ & 8 & $4.9 \%$ \\
\hline $\begin{array}{l}\text { Mano / } \\
\text { Muñeca - } \\
\text { Izquierda }\end{array}$ & 17 & $10.4 \%$ & 7 & $4.3 \%$ & 39 & $23.8 \%$ & 10 & $6.1 \%$ & 58 & $35.4 \%$ & 7 & $4.3 \%$ & 24 & $14.6 \%$ & 2 & $1.2 \%$ \\
\hline $\begin{array}{l}\text { Mano / } \\
\text { Muñeca - } \\
\text { Derecha }\end{array}$ & 19 & $11.6 \%$ & 5 & $3.0 \%$ & 46 & $28.0 \%$ & 3 & $1.8 \%$ & 64 & $39.0 \%$ & 1 & $0.6 \%$ & 26 & $15.9 \%$ & 0 & $0.0 \%$ \\
\hline
\end{tabular}

Descripción: *Si - Presenta sintomatología.

*No - No presenta sintomatología.

Fuente: Autores.

De forma global los datos del género femenino (Figura 2), evidencian que el rango de edad entre 35 a 44 años presenta mayores porcentajes de percepción de problemas musculoesqueléticos también en los rangos de edad de 25-34 años y 35-44 años. En ocho de las nueve preguntas del cuestionario planteado. El rango de edad de 35 a 44 años evidencia los más altos porcentajes siendo las dolencias más prevalentes en: mano muñeca derecha $39 \%$, cuello $38.4 \%$, columna lumbar $37.8 \%$, mano muñeca izquierda $35.4 \%$, el segundo rango de edad con dolencias más prevalentes es el de 25-34 años evidenciando en: cuello y mano muñeca derecha con el $28 \%$, columna lumbar $26.8 \%$, y mano muñeca izquierda $23.8 \%$. 
Figura 2

Percepción de sintomatología musculoesquelética - Género Femenino.

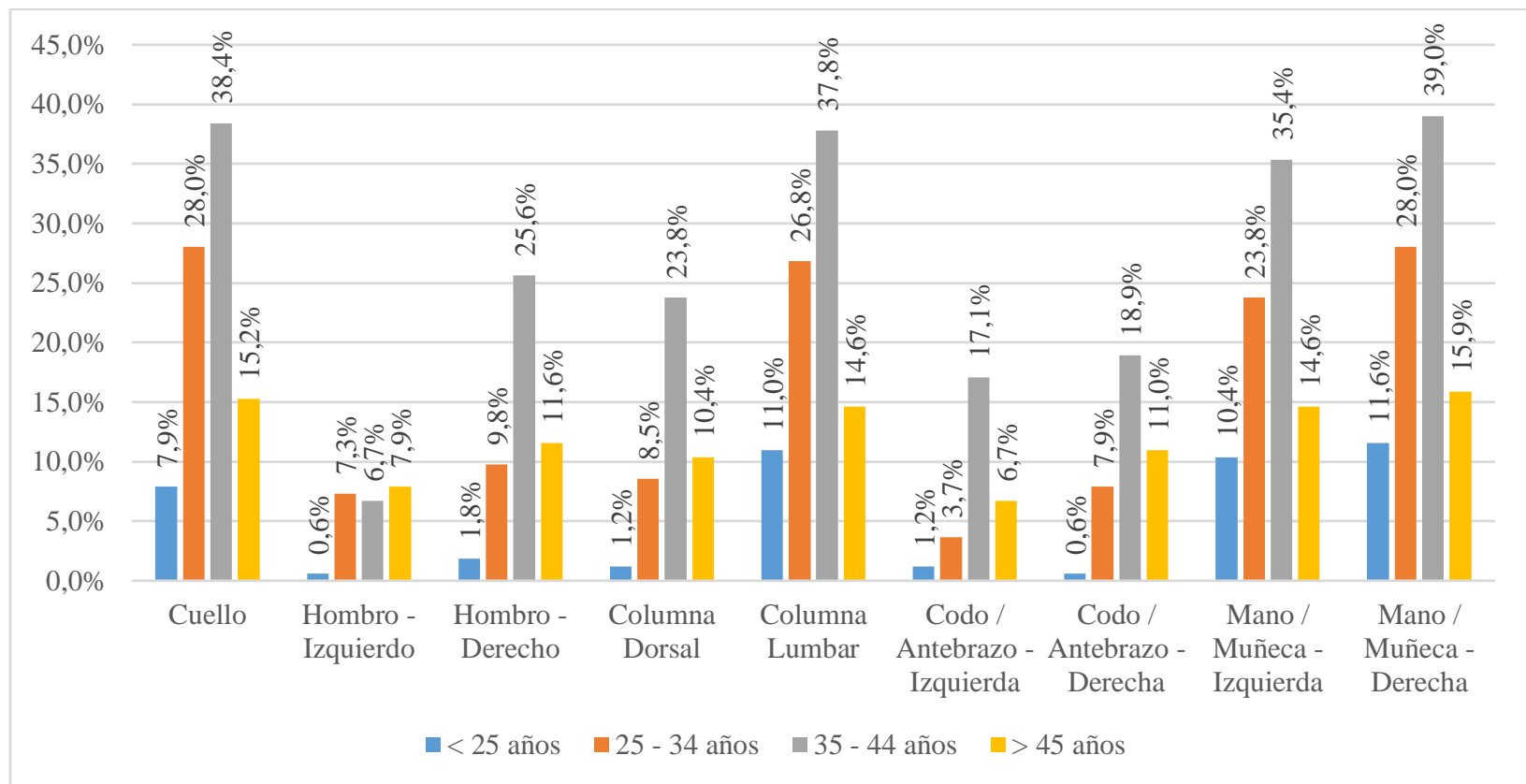

Fuente: Autores.

La evidencia de los datos globales (Figura 3) de comparación entre el género masculino $\left(\mathrm{n}_{1}=104\right)$ y el género femenino $\left(\mathrm{n}_{2}=164\right)$, reflejan que los segmentos corporales con mayor percepción de trastornos musculoesqueléticos superan el 75\% siendo: mano muñeca derecha con el 95.2\% $\left(\mathrm{n}_{1}=104\right)$ en el género masculino y el $94.5 \%\left(\mathrm{n}_{2}=164\right)$ en el género femenino, en columna lumbar alcanza el $91.3 \%\left(n_{1}=104\right)$ en género masculino y el $90.2 \%\left(n_{2}=164\right)$ en género femenino, en el segmento corporal cuello se evidencia que el género femenino alcanza el 89.6\% $\left(\mathrm{n}_{2}=164\right)$ frente al $87.5 \%\left(\mathrm{n}_{1}=104\right)$ del género masculino, los datos tabulados de mano muñeca izquierda refleja el $84.1 \%\left(\mathrm{n}_{2}=164\right)$ en el género femenino y el $76 \%\left(\mathrm{n}_{1}=104\right)$ en el género masculino. Los segmentos corporales inferiores al $50 \%$ de cada muestra analizada por género son: hombros derecho e izquierdo, columna dorsal; $y$, codo antebrazo derecho e izquierdo respectivamente. 
Figura 3

Comparativa de Sintomatología musculoesquelética por segmentos corporales y género.

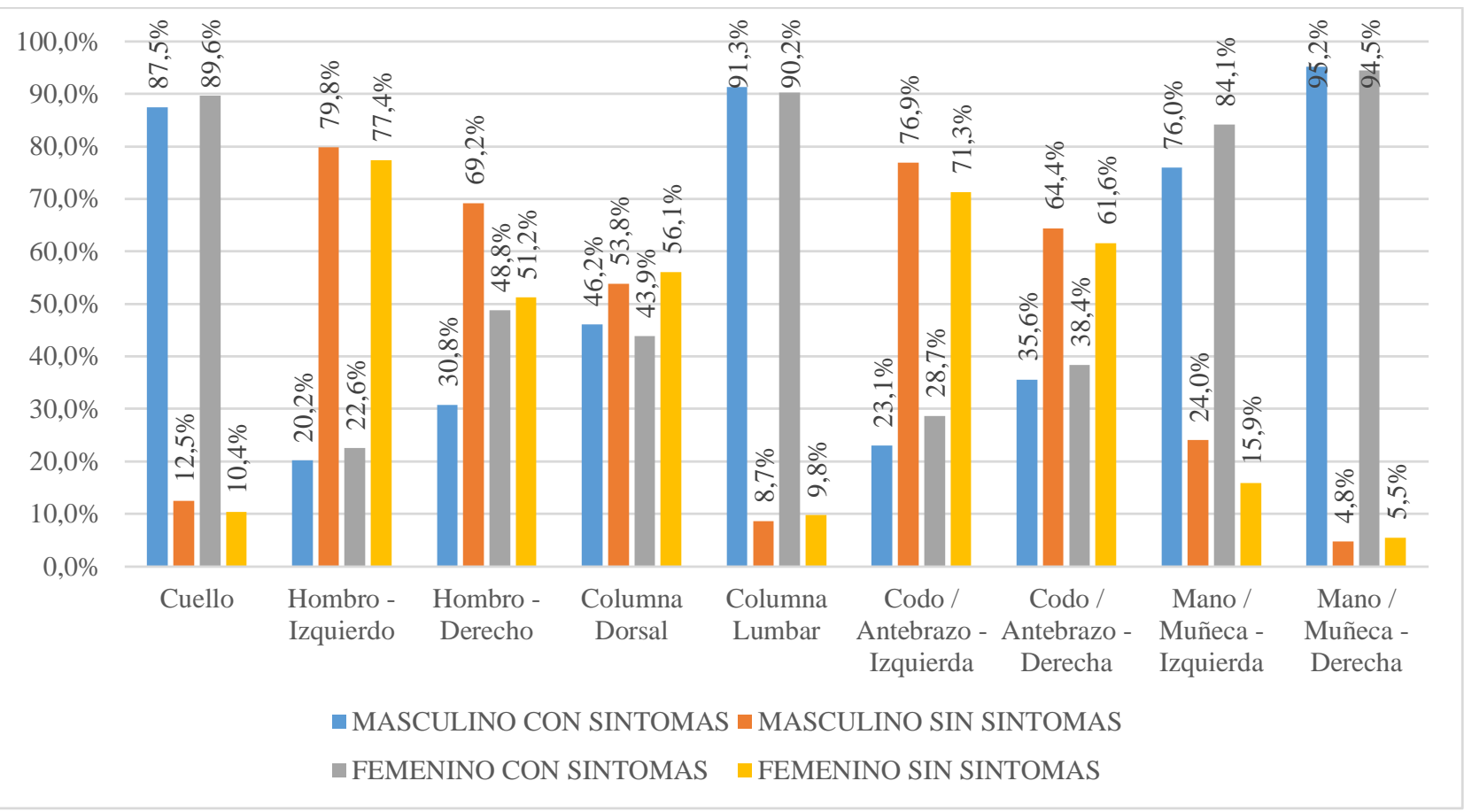

Fuente: Autores.

\section{Conclusiones}

El análisis de la primera variable sociodemográfica correspondiente al género se evidencia que el $61.2 \%(\mathrm{~N}=268 ; 164)$ son de género femenino y el $38.8 \%(\mathrm{~N}=268 ; 104)$ corresponde al género masculino.

En el género masculino y femenino se evidencia en forma global, que en el rango de edad de 35 a 44 años demuestran mayores porcentajes de percepción de problemas musculoesqueléticos en los segmentos corporales mano muñeca derecha, cuello, columna lumbar y mano muñeca izquierda.

Los hallazgos de este estudio evidencian que los datos globales de comparación entre los géneros masculino $\left(\mathrm{n}_{1}=104\right)$ y el género femenino $\left(\mathrm{n}_{2}=164\right)$, en la cual los segmentos corporales con mayor percepción de trastornos musculoesqueléticos y que superan el $75 \%$ de cada muestra son: mano muñeca derecha con el $95.2 \%\left(\mathrm{n}_{1}=104\right)$ en el género masculino y el $94.5 \%\left(\mathrm{n}_{2}=164\right)$ en el género femenino, en columna lumbar se alcanza el $91.3 \%\left(\mathrm{n}_{1}=104\right)$ en género masculino y el 90.2\% $\left(\mathrm{n}_{2}=164\right)$ en el género femenino, el segmento corporal cuello evidencia que el género femenino alcanza el $89.6 \%\left(\mathrm{n}_{2}=164\right)$ frente al $87.5 \%\left(\mathrm{n}_{1}=104\right)$ del género masculino, los datos tabulados de mano muñeca izquierda alcanza el $84.1 \%\left(\mathrm{n}_{2}=164\right)$ en género femenino y el $76 \%$ $\left(n_{1}=104\right)$ en género masculino. Los segmentos corporales inferiores al $50 \%$ de cada muestra 
analizada por género son: hombros derecho e izquierdo, columna dorsal; y, codo antebrazo derecho e izquierdo respectivamente.

Varios estudios exponen que la mayor prevalencia de dolor entre los empleados administrativos de una empresa pertenece a las partes del cuello y la espalda baja. (Loghmani et al., 2013). Un estudio holandés señala que el $28 \%$ de la población reportó dolor o rigidez en el cuello, espalda baja y muñecas. (Chiung et al., 2009), por lo que los resultados obtenidos se relacionan en referencia a las afecciones identificadas. La prevalencia del dolor en la columna lumbar, cuello y muñeca señala la presencia de peligros en actividades laborales relacionadas a un factor de riesgo ergonómico que afecta la salud de los trabajadores, y que puede llegar a convertirse en una enfermedad laboral por lo que la intervención en mediciones y rediseño a los puestos de trabajo es de vital importancia.

Con el presente estudio de investigación basados en el Cuestionario Nórdico Estandarizado de Síntomas Musculoesqueléticos de Kuorinka, se concluye que a través de la percepción se logra identificar el inicio de un posible trastorno musculoesquelético para poder plantearse medidas de prevención y control ergonómicas en los puestos de trabajo administrativos, anticipándose a posibles enfermedades ocupacionales pudiendo tener consecuencias y afectaciones a los trabajadores e incrementando el absentismo laboral. Los datos obtenidos reflejan la aseveración de organismos y centros de investigación de reconocido prestigio que las mayores molestias se producen en la columna lumbar, cuello y síndrome de túnel carpiano que pertenece al segmento corporal de mano muñeca derecha e izquierda analizado. Los autores con la presente investigación evidencian el nivel basal de lo que está ocurriendo en empresas de nuestro país, dejando las puertas abiertas para próximas investigaciones acerca de la gestión de la ergonomía y su relación directa con la salud de los trabajadores y la productividad de las organizaciones.

\section{Referencias Bibliográficas}

Alaca, N., Safran, E., Karamanlargil, A., \& Timucin, E. (2019). Translation and cross-cultural adaptation of the extended version of the Nordic musculoskeletal questionnaire into Turkish. Journal of Musculoskeletal Neuronal Interactions, 19(4), 472-481.

Arenas-Ortiz, L., \& Cantú-Gómez, Ó. (2013). Factores de riesgo de trastornos músculoesqueléticos crónicos laborales. Medicina Interna de Mexico, 29(4), 370-379.

Bellorín, M., Sirit, Y., Rincón, C., \& Amortegui, M. (2007). Síntomas músculo esqueléticos en trabajadores de una empresa de construcción civil. Salud de Los Trabajadores, 15(2), 8998.

Bosman, L. C., Twisk, J. W. R., Geraedts, A. S., \& Heymans, M. W. (2020). Effect of Partial Sick Leave on Sick Leave Duration in Employees with Musculoskeletal Disorders. Journal of Occupational Rehabilitation, 30(2), 203-210. https://doi.org/10.1007/s10926-019-09864$\mathrm{Z}$

Chiung, C., Hwang, Y. S., \& Cherng, R. J. (2009). Musculoskeletal Symptoms and Associated Risk Factors Among Office Workers With High Workload Computer Use. Journal of Manipulative and Physiological Therapeutics, 35(7), 534-540. 
https://doi.org/10.1016/j.jmpt.2012.07.004

Dimate, A. E., Rodríguez, D. C., \& Rocha, A. I. (2017). Percepción de desórdenes musculoesqueléticos y aplicación del método RULA en diferentes sectores productivos: una revisión sistemática de la literatura. Revista de La Universidad Industrial de Santander. Salud, 49(1), 57-74. https://doi.org/10.18273/revsal.v49n1-2017006

Gómez Rodríguez, R., Díaz Pulido, B., Gutiérrez Ortega, C., Sánchez Sánchez, B., \& Torres Lacomba, M. (2020). Cultural adaptation and psychometric validation of the standardised nordic questionnaire Spanish version in musicians. International Journal of Environmental Research and Public Health, 17(2), 1-10. https://doi.org/10.3390/ijerph17020653

INSHT. (2017). Encuesta Nacional de Condiciones de Trabajo. $20156^{\mathrm{a}}$ EWCS - España Autor. Instituto Nacional de Seguridad e Higiene En El Trabajo. https://www.insst.es/

Iti, J., Nigudgi, S., \& Reddy, S. (2016). Assessment of musculoskeletal disorders by standardized nordic questionnaire among computer engineering students and teaching staff of Gulbarga city. International Journal of Community Medicine and Public Health, 3(3), 668-674. https://doi.org/10.18203/2394-6040.ijcmph20160630

Kuorinka, I., Jonsson, B., Kilbom, A., Vinterberg, H., Biering-Sørensen, F., Andersson, G., \& Jørgensen, K. (1987). Standardised Nordic questionnaires for the analysis of musculoskeletal symptoms. Applied Ergonomics, 18(3), 233-237. https://doi.org/10.1016/0003-6870(87)90010-X

Kwaku Essien, S., Trask, C., \& Dosman, J. (2016). Investigating the association between lower extremity and low back symptoms among Saskatchewan farmers using the Standardized Nordic Questionnaire. International Journal for the Study of the Spine Publish, 30(8), 56. https://doi.org/10.1097/BRS.0000000000002113

Loghmani, A., Golshiri, P., Zamani, A., Kheirmand, M., \& Jafari, N. (2013). Musculoskeletal symptoms and job satisfaction among office-workers: a cross-sectional study from Iran. Acta Medica Academica, 42(1), 46-54. https://doi.org/10.5644/ama2006-124.70

Martínez, M. M., \& Alvarado Muñoz, R. (2017). Validación del Cuestionario Nórdico Estandarizado de Síntomas Musculoesqueléticos para la población trabajadora chilena, adicionando una escala de dolor. Revista de Salud Pública, 21(2), 43. https://doi.org/10.31052/1853.1180.v21.n2.16889

Mendinueta Martínez, M., \& Herazo Beltrán, Y. (2014). Percepción de molestias musculoesqueléticas y riesgo postural en trabajadores de una institución de educación superior Perception musculoskeletal discomfort and postural risk among employees of a higher education institution. Salud Uninorte, 30(2), 170-179.

Namnik, N., Negahban, H., Salehi, R., Shafizadeh, R., \& Tabib, M. S. (2016). Validity and reliability of Persian version of the Specific Nordic questionnaire in Iranian industrial workers. Work, 54(1), 35-41. https://doi.org/10.3233/WOR-162268

Nazish, N., Charles, M. J., \& Kumar, V. (2020). Prevalence of Musculoskeletal Disorder among House Wives and Working Women. 10(February), 215-222.

Oliveira, R. G. de, Pires-Oliveira, D. A. de A., Oliveira, L. C. de, Remonte, A. K., Garcia, S. C., Araújo, C. C. de, Jassi, F. J., Ramos, W. L., Venâncio, P. E. M., Teixeira, J., \& Oliveira, R. F. de. (2017). Translation, transcultural adaptation and validation of the Nordic questionnaire for the evaluation of low back pain in Brazilian adolescents. Manual Therapy, Posturology \& Rehabilitation Journal, 15(November), 1-6. https://doi.org/10.17784/mtprehabjournal.2017.15.505

OMS. (2004). La organización del trabajo y el estrés. Serie Protección de La Salud de Los

Esta obra se comparte bajo la licencia Creative Common Atribución-No Comercial 4.0 International (CC BY-NC 4.0) Revista de la Universidad Internacional del Ecuador. URL: https://www.uide.edu.ec/ 
Trabajadores $N^{\circ}$ 3, 3(1729-3510), 37. https://doi.org/ISBN 9243590472

Orhan, K., \& Memon, A. (2019). Introductory Chapter_Work-Related Musculoskeletal Disorders _IntechOpen. https://doi.org/10.5772 / intechopen.85479

Qureshi, A., Manivannan, K., Khanzode, V., \& Kulkarni, S. (2019). Musculoskeletal disorders and ergonomic risk factors in foundry workers. International Journal of Human Factors and Ergonomics, 6(1), 1-17. https://doi.org/10.1504/IJHFE.2019.099579

Rasha, A., Amir, M., Elsayed, S. E., \& Dawood, R. S. (2019). Work Related Musculoskeletal Disorders Among Egyptain Physical Therapists and Years of Experience. SVUInternational Journal of Physical Therapy and Science, 1(1), 1-7.

Widanarko, B., Legg, S., Devereux, J., \& Stevenson, M. (2014). The combined effect of physical, psychosocial/organisational and/or environmental risk factors on the presence of workrelated musculoskeletal symptoms and its consequences. Applied Ergonomics, 45(6), 1610-1621. https://doi.org/10.1016/j.apergo.2014.05.018

Zamanian, Z., Norouzi, F., Esfandiari, Z., Rahgosai, M., \& Hasan, F. (2017). Assessment of the prevalence of musculoskeletal disorders in nurses. Yasuj University of Medical Sciences Journal (YUMSJ), 21(10), 976-986. 